\title{
PERSPECTIVE
}

\section{The ACA's 65th Birthday Challenge: Moving from Medicaid to Medicare}

\author{
Chima D. Ndumele, Ph.D. ${ }^{7}$, Benjamin D. Sommers, M.D., Ph.D. ${ }^{2,3}$, and \\ Amal N. Trivedi, M.D., M.P.H. ${ }^{4,5}$ \\ 'Department of Health Policy and Management, Yale School of Public Health, New Haven, CT, USA; '2Department of Health Policy and \\ Management, Harvard School of Public Health (B.D.S), Boston, MA, USA; ${ }^{3}$ Brigham and Women's Hospital (B.D.S.), Boston, MA, USA; ${ }^{4}$ Department of \\ Health Services, Policy and Practice, Brown University School of Public Health (A.N.T), Providence, RI, USA; ${ }^{5}$ Providence VA Medical Center (A.N.T.), \\ Providence, RI, USA.
}

The Affordable Care Act (ACA) expanded Medicaid to millions of low-income near-elderly Americans, facilitating access to health care services, but did not change income eligibility for Medicaid for those 65 years and older. Therefore, following the ACA's coverage expansion, many newly-insured older enrollees will face a complex insurance transition on their 65th birthday: they will lose Medicaid coverage and transition from Medicaid to Medicare as their primary insurer. This transition in primary health insurance coverage includes changes to benefits, patient cost-sharing, and provider reimbursement, which could have profound consequences on the use of health services and associated health outcomes for low-income seniors. Using data from 2012, we estimate that 1.6 million current Medicaid beneficiaries and an additional 1.6 to 2.9 million low-income individuals who will gain Medicaid coverage under the ACA will be likely to make this transition in the next decade. Primary care physicians and policymakers can help mitigate the potential consequences of this insurance transition by preparing patients for Medicare's more restrictive insurance coverage, encouraging patients to sign up for available low-income subsidies, and understanding how the loss of Medicaid coverage impacts out-of-pocket costs.

J Gen Intern Med 30(11):1704-6

DOI: $10.1007 / \mathrm{s} 11606-015-3328-0$

(c) Society of General Internal Medicine 2015

$\mathrm{T}$ o illustrate the consequences of this insurance transition, we discuss a hypothetical patient with Medicaid coverage who loses eligibility on her 65th birthday. Ms. Jones is a 64year-old woman with diabetes, hyperlipidemia, and depression, living on an annual income of just over $\$ 13,000$, who has struggled for years to find affordable health insurance. In 2014, she gained Medicaid coverage under the Affordable Care Act (ACA). Policymakers designed the ACA to assist

Received January 7, 2015

Revised March 18, 2015

Accepted March 25, 2015

Published online April 23, 2015 patients just like Ms. Jones, who previously would not have qualified for Medicaid and could not afford purchasing insurance on her own. It is indeed a notable policy accomplishment. But the story of Ms. Jones' search for affordable insurance coverage is not over. Her 65th birthday will bring an unexpected and potentially unwelcome surprise: she will lose her newly-acquired Medicaid coverage and transition to Medicare.

The ACA expands Medicaid in participating states by increasing the income threshold for eligibility to $138 \%$ of the Federal Poverty Level (FPL) and no longer requiring persons to belong to a category of eligibility such as a disability, pregnancy, or parent of a dependent child. The ACA's Medicaid expansions have the potential to increase continuous health insurance coverage, reduce catastrophic health spending, and improve health outcomes for low-income uninsured Americans, while preserving access to care for those already covered by Medicaid. ${ }^{1,2}$ Nonetheless, unforeseen challenges often accompany substantial health policy changes.

While the ACA expanded Medicaid's income eligibility for non-elderly adults, the federal minimum standard for Medicaid eligibility for those 65 and older remained unchanged at approximately $75 \%$ of the FPL. Though some states have increased their Medicaid eligibility for the elderly to higher levels, the majority of states limit Medicaid to individuals below $100 \%$ of the FPL and none match the ACA's $138 \%$ cutoff for younger adults.

Consider again our hypothetical patient, Ms. Jones. Her annual income is $\$ 13,350$ (115\% of FPL) and her assets are just under $\$ 5000$. On her 65th birthday, she will lose Medicaid eligibility and acquire Medicare. As a new Medicare enrollee, she must choose traditional Medicare or Medicare Advantage, identify a prescription drug plan that offers preferred coverage for her current medications, and determine whether she qualifies for a financial assistance program for low-income Medicare beneficiaries. While many of the complexities associated with navigating the Medicare program are challenging for many new beneficiaries and have been even prior to the ACA, choosing a Medicare plan may prove especially difficult for Medicaid enrollees with little experience navigating health insurance markets. Ideally, Ms. Jones would select an option 
that minimizes her spending on premiums and copayments and includes her current primary care and specialist providers in the plan's contracted network. But prior evidence suggests seniors often fail to select the optimal insurance option, with greater complexity of choices eroding the quality of decisionmaking. ${ }^{3}$

Ms. Jones' income and assets disqualify her from subsidies to help pay Part B copayments and deductibles, which are restricted to individuals or couples making less than $\$ 11,916$ or $\$ 15,972$ a year, respectively, and assets lower than $\$ 11,000$. Although she qualifies for coverage of the Part B premium and Part D subsidies, available to individuals making less than $100 \%$ FPL and $135 \%$ FPL, respectively, she must be aware of these programs and take the steps to apply for them. Of those who are eligible and not automatically assigned, take-up of Part D low-income subsidies and Part B premium subsidies remains well under $50 \%$.

Medicare's more restrictive insurance benefits may be particularly daunting for people like Ms. Jones. Medicaid programs are generally prohibited from imposing significant outof-pocket payments requirements, with regulations limiting cost-sharing to no more than $5 \%$ of annual income. Unlike Medicaid, Medicare does not limit annual out-of-pocket spending. Traditional Medicare enrollees without other supplemental coverage must pay a large inpatient deductible (over $\$ 1200$ in 2014) and $20 \%$ of the Medicare-approved amount for nonpreventive outpatient services (in addition to the $\$ 105$ monthly premium for Part B). Medicare Advantage plans also typically include substantial copayments and require a monthly premium. Medigap policies, which average over \$2000 dollars in annual premiums, would likely be unaffordable for Ms. Jones. Annual out-of-pocket costs for non-disabled Medicaid recipients have been estimated to be between $\$ 210$ and \$560, in stark contrast to annual out-of-pocket spending of $\$ 4,734$ among Medicare beneficiaries in 2010. ${ }^{5-7}$ Finally, some of her current medications may not be in the preferred tier of available Part D plans, which may erode adherence to her oral hypoglycemic, lipid-lowering, and antidepressant therapy. ${ }^{8}$

How many Medicaid enrollees might experience this change in coverage on their 65th birthday? We analyzed nationally representative data from the American Community Survey from before the ACA's Medicaid expansions to identify adults ages 55-64 years who may be at-risk for losing Medicaid coverage. We assigned each individual a primary type of coverage if they reported more than one type, using a hierarchy of Medicare, then Medicaid, employer sponsored insurance, non-group coverage, and other insurance. This means that dual-eligibles were treated as having Medicare (which is in fact the primary payer for this population). Figure 1 describes our results both for the full 55-64 year age group and for the subset with family incomes below $138 \%$ of the Federal Poverty Level, which qualifies them for Medicaid coverage in participating states under the ACA.

We estimate that, as of 2012, $6.6 \%$ of all adults in this age range and $23 \%$ among the low-income group (representing

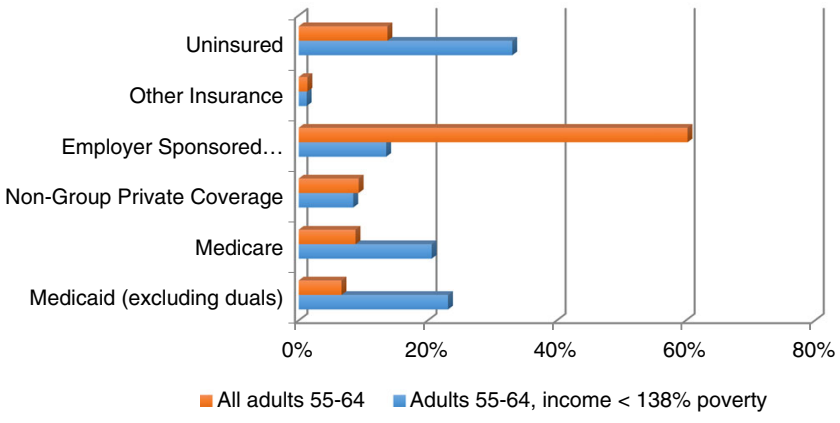

Figure 1 Pre-ACA coverage for adults ages 55-64, by income level, authors' analysis of data from the 2012 American Community Survey $(n=2,620,739)$. Coverage was assigned using a health insurance hierarchy, so each person has one type of primary coverage. The hierarchy was Medicare, Medicaid, ESI, Non-Group, Other. Family income was determined by the health insurance unit, which consists of an adult, his/her spouse, and dependent children. These results include all U.S. residents. Sensitivity analyses examining only U.S. citizens showed similar results.

just under 1.6 million adults) had Medicaid but not Medicare. This group represents the pre-ACA portion of near-elderly adults likely to make a Medicaid-to-Medicare transition at age 65. However, the ACA will add millions more to this group. The Medicaid expansion is most likely to enroll lowincome individuals who were previously uninsured (33\%) or had non-group coverage $(8 \%)$, which corresponded to an additional 2.6 million adults in 2012. While some of these individuals may meet criteria to remain in Medicaid upon turning 65 , nearly all of them would be expected to transition their primary insurance from Medicaid to Medicare within the next decade, assuming all states eventually participate in the Medicaid expansion. If we limit our analysis to the 28 states and Washington D.C. that have chosen to expand Medicaid as of February 2015, we estimate than there are still an additional 1.6 million adults likely to be newly eligible for Medicaid under the ACA and then transition to Medicare in the next decade.

How might policymakers mitigate the potential adverse impacts of this transition? One simple - but potentially costly and controversial solution - would be raising Medicaid's income eligibility for those 65 and older to match or at least close the gap with the ACA's $138 \%$ FPL cutoff for younger adults.

Less dramatic options also exist that may be more politically practical. First, the Centers for Medicare and Medicaid Services (CMS) and state Medicaid programs could prospectively identify all older enrollees who are at-risk for losing Medicaid coverage, and provide outreach to assist their transition to Medicare. Many states successfully used navigators to help consumers choose plans in the health insurance marketplaces during the first open-enrollment period of the ACA. A similar strategy may optimize the transitions to Medicare by identifying options that maximize continuity and minimize out-of-pocket expenses. Second, states could notify former Medicaid recipients and facilitate their enrollment in financial 
assistance programs that subsidize Part B premiums and Medicare copayments. CMS has successfully implemented this approach among other groups to bolster take-up of Part D lowincome subsidies among populations who are not auto-enrolled. Finally, policymakers could expand the eligibility for such assistance programs for older adults who no longer qualify for Medicaid eligibility. This change would benefit many patients like Ms. Jones, who have incomes that currently disqualify them from obtaining financial assistance with Medicare's cost-sharing.

These issues have important implications for general internists who care for older adults with Medicaid coverage. First, many such patients may be unaware that they are at risk for losing their Medicaid coverage. Care management teams can prepare older Medicaid enrollees, particularly those newly insured via the ACA, for the likely consequences of their transition to Medicare and anticipate the unique challenges this insurance transition poses to low-income patients. Second, since Medicare covers fewer services and includes higher cost-sharing than does Medicaid, clinicians and case-managers should be vigilant to monitor patients out-of-pocket costs to ensure they are not foregoing important medications and outpatient care. Finally, clinicians should be aware that patients who transition from Medicaid to Medicare may be more likely to change physicians, since a higher proportion of physicians accept Medicare coverage and patients enrolled in Medicare Advantage plans may have restrictions on their choice of physicians.

The ACA is expanding Medicaid insurance in participating states, with the potential to enhance coverage and access to care for millions of low-income adults. But a new challenge will accompany coverage gains for those who lose Medicaid at age 65. Vigilance by policymakers and clinicians for those who transition from Medicaid to Medicare coverage can maximize the benefits of the ACA's Medicaid expansion and avoid unintended adverse effects for vulnerable low-income seniors.
Acknowledgements: Dr. Sommers' work on this project was supported by grant number K02HSO21291 from theAgency for Healthcare Research and Quality (AHRQ). Dr. Sommers also serves as an advisor in the Office of the Assistant Secretary for Planning and Evaluation at the Department of Health and Human Services (HHS), but the views presented here do not represent HHS or AHRQ.

Conflicts of Interest: The authors declare that they do not have a conflict of interest.

Corresponding Author: Chima D. Ndumele, Ph.D.; Department of Health Policy and ManagementYale School of Public Health, 60 College St, New Haven, CT 06520, USA (e-mail: chima.ndumele@yale.edu).

\section{REFERENCES}

1. Ndumele CD, Mor V, Allen S, et al. Effect of expansions in state Medicaid eligibility on access to care and the use of emergency department services for adult Medicaid enrollees. JAMA Intern Med. 2014;174(6):920-6.

2. Sommers BD, Baicker K, Epstein AM. Mortality and access to care among adults after state Medicaid expansions. N Engl J Med. 2012;367(11):102534 .

3. McWilliams JM, Afendulis CC, McGuire TG, et al. Complex Medicare advantage choices may overwhelm seniors-especially those with impaired decision making. Health Aff (Millwood). 2011;30(9):1786-94.

4. Shoemaker JS, Davidoff AJ, Stuart B, et al. Eligibility and take-up of the Medicare Part D low-income subsidy. Inquiry. 2012;49(3):214-30.

5. Nowak SA, Eibner C, Adamson DM, et al. Effects of the Affordable Care Act on Consumer Health Care Spending and Risk of Catastrophic Health Costs. Rand Corporation Report. 2013; available at http:// www.rand.org/content/dam/rand/pubs/research_reports/RR300/ RR383/RAND RR383.pdf

6. Cubanski J, Swwope C, Damico A et al. How Much is Enough? Out-of Pocket Spending Among Medicare Beneficiaries: A Chartbook. Kaiser Family Foundation Report. 2014; avaialble at http://kff.org/health-costs/report/ how-much-is-enough-out-of-pocket-spending-among-medicare-beneficiaries-a-chartbook/

7. Broaddus $\mathbf{M}$ and $\mathbf{K u} \mathbf{L}$. Out of Pocket Medical Expenses for Medicaid Beneficiaries are Substantial and Growing. Center on Budget and Policy Priorities Report. 2005; available at http://www.cbpp.org/cms/? $\mathrm{fa}=$ view\&id $=320$

8. Maciejewski ML, Bryson CL, Perkins M, et al. Increasing copayments and adherence to diabetes, hypertension, and hyperlipidemic medications. Am J Manag Care. 2010;16(1):e20-34. 